\title{
Integration of Software Defined Radio and Add-on Board into Digital Communication Education with Hands-on Applications
}

\author{
Emrah GENC ${ }^{* 1}$, Cagdas TUNA ${ }^{2}$, Safak SARAYDEMIR ${ }^{2}$
}

\author{
Accepted $3^{\text {rd }}$ September 2016
}

\begin{abstract}
Owing to the theory depend heavily on mathematical models in communication lessons, instructors find students lost in all the equations and notations that given. Setting a lab component for students to put together the theory with practical implementations is not very easy in many times because it may cost a large number of equipment that is not low-priced. Yet there are still some solutions which reduce the cost in designing a real-life communication laboratory and effective in teaching. We present the usage of NI-USRP Software Defined Radio (SDR) and Emona DATEx Add-on Board on digital modulation techniques that take part in communication engineering education. Most commonly used digital modulation techniques; Amplitude Shift Keying (ASK), Phase Shift Keying (PSK) and Frequency Shift Keying (FSK) are analyzed in both devices. Both modulations and demodulations of mentioned techniques are done by experimentally.
\end{abstract}

Keywords: Software Defined Radio, Communication Engineering Education, Digital Modulation Techniques, USRP, DATEx.

\section{Introduction}

In general most of the students at communication engineering start learning communication systems with the theory and the principles. However learning the theory and principles solely does not help students to design communication systems properly. They need to acquire experimental skills beside the theoretical domain. In order to bridge this gap instructors use several equipments such as function generators, oscilloscopes, spectrum analyzers that is not affordable many times.

With the development of microprocessors and software technology, traditional equipments began to transform into computer hosted devices which are low-cost, flexible and multifunctional. As an example, SDR, offers large number of tools to teach signals and communications [1]. Limits of traditional equipments, students being remained at the level of pre-digital area and the motivation for SDR based communication education is stated clearly in [2]. SDR refers that analog-to-digital (A/D) conversion is performed at the antenna connector, with all following signal processing (upconversion and/or downconversion, intermediate-frequency (IF) filtering, demodulation, etc.) performed in reconfigurable software using digital signal processing (DSP) techniques [3].

There are several examples of SDR based education courses. Domain analyses such as time, frequency, joint time-frequency, code, and modulation discussed in [4]. M-ary PSK modulation and demodulation implemented in [5]. AM implementation presented in [6]. Five week communication applications programme discussed in [7].Therefore, multi-purpose usage of SDR in communication lessons will be educatory and preliminary

${ }^{1}$ Turkish Military Academy, Defense Sciences Institute, Ankara, Turkey

${ }^{2}$ Turkish Military Academy, Department of Electrical Engineering, Ankara, Turkey

*Corresponding Author: Email: egenc@kho.edu.tr

Note: This paper has been presented at the $3^{\text {rd }}$ International Conference on Advanced Technology \& Sciences (ICAT'16) held in Konya (Turkey), September 01-03, 2016. for real world.

In this study's first part we use NI-USRP 2922 supporting frequency range of $400 \mathrm{MHz}$ to $4.4 \mathrm{GHz}$ with SBX daughterboard. It's omni-directional vertical antenna provides 3 $\mathrm{dBi}$ gain for two-way high bandwidth communication. A Gigabit Ethernet connectivity provide data streaming to host processors enables simultaneously sending up to $50 \mathrm{MHz}$ RF signal in and out of the USRP device [8].

Another way of using computer hosted device in communication education is Emona DATEx. By using it students can find the opportunity to experiment the real world signals in lessons.

The Emona DATEx, Digital Analog Telecommunications Experimenter, is an add-on which could serve as a basic training board in various concepts of communication lessons [9]. This add-on is fully integrated with the NI ELVIS II platform and is controllable through the NI LabVIEW development environment. All DATEx knobs and switches are manually controllable or programmable from LabVIEW.

The NI ELVIS II is an all-in-one electronics workstation that performs the functions of instruments which are conventionally found in an electrical engineering laboratory. The NI ELVIS II holds a removable prototyping board on which developers can build electrical circuits which connect the different ELVIS's instruments to achieve a particular experimental goal.

In communication education, examples of DATEx usage limited. Practical aspects of Direct Sequence Spread Spectrum (DSSS) communication discussed in [10]. AM/FM/BPSK signal generating experiments over the internet is presented in [11]. Time and frequency domain demonstrations discussed in [12].

\section{ASK Modulation Applications}

ASK is a form of amplitude modulation that the amplitude of the carrier wave is shifted proportionally to the amplitude of the digital signal. For a binary message sequence there are two levels; a binary " 0 " is one amplitude and a binary " 1 " is another amplitude. We can formulate ASK as;

$$
s(t)=A m(t) \cos (2 \pi f c t) \quad 0 \leq t \leq T
$$


where $A$ is a constant, $m(t)=1$ or $0, f c$ is the carrier frequency, and $T$ is the bit duration.

\subsection{ASK Implementation with USRP}

USRP Hardware Driver works on a variety of programming languages, operating systems, and development environments such as Linux, Windows, Mac and can be used with numerous frameworks such as GNU Radio, LabVIEW, Simulink, OpenBTS, OSSIE, Redhawk etc. In this study all applications done with LabVIEW because USRP devices that we have supported with licensed LabVIEW Framework.

The basic building block of LabVIEW is the virtual instrument (VI). Each VI consists of a block diagram and a front panel. The block diagram describes the functionality of the VI, while the front panel is a top level interface to the VI. Fig. 1 shows front panel of ASK application.

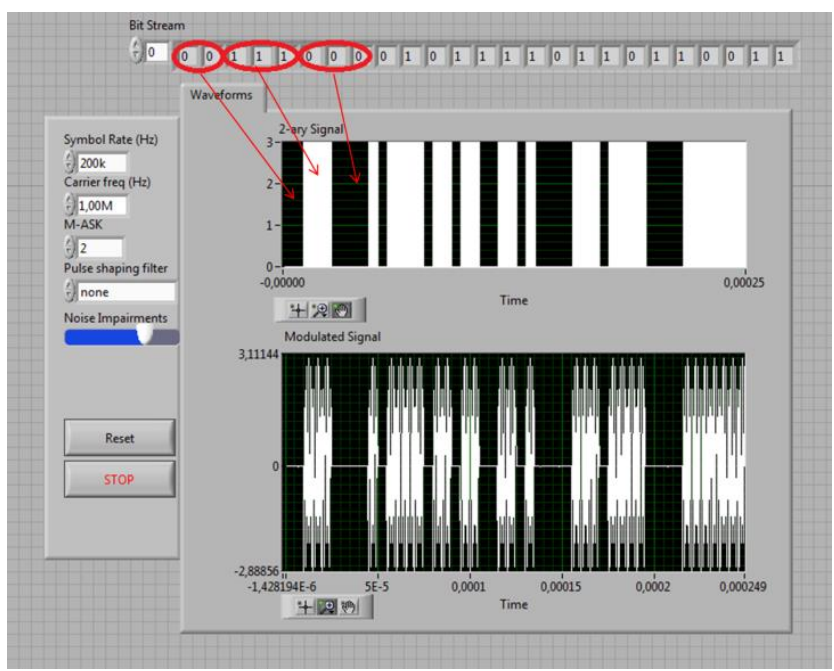

Figure 1. Front Panel of ASK Application.

At the top of the front panel bit stream represents digital data that is randomly produced. At the bottom of the front panel ASK signal is located. While generating ASK signal, all we have to do is to connect the subVIs to each other in an order and feed them with the wires properly. But the theories like Nyquist theorem is crucial while establishing symbol rate values via carrier frequency. Moreover for the getting pure ASK, sampling rate and noise impairment values must be chosen punctiliously. In Fig. 2 block diagram of the ASK application is shown.

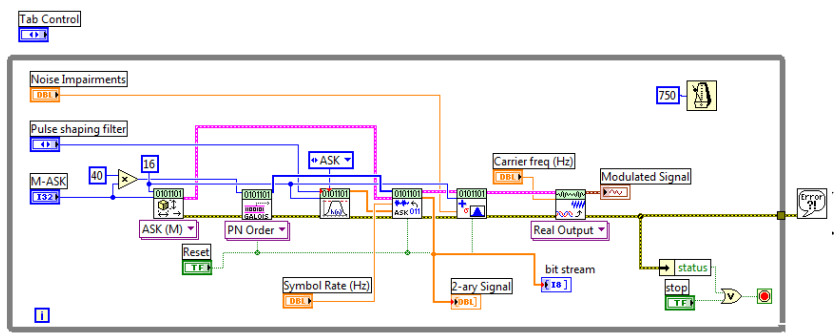

Figure 2. Block Diagram of ASK Application.

\subsection{ASK Implementation with DATEx}

In DATEx implementation we use Sequence Generator module for the digital data. $100 \mathrm{kHz}$ master signal is quite appropriate for the carrier frequency because in ideal ASK communication system, we want the carrier frequency to be much higher than the bit rate of the digital data. Generating ASK signal is provided by
Dual Analog Switch module. In Fig. 3 modulation (Dotted) and demodulation (Lined) scheme of ASK is shown.

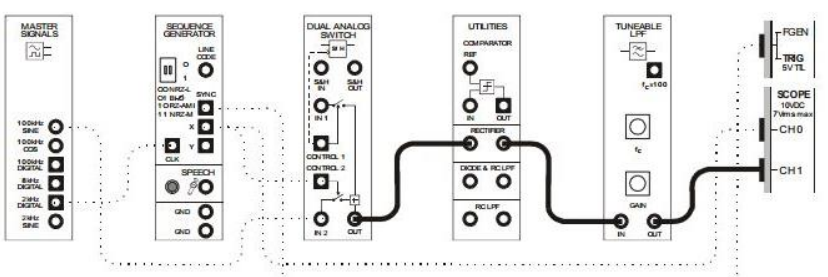

Figure 3. ASK Modulation and Demodulation Scheme.

In demodulation part of DATEx implementation for getting an envelope detector, rectifier on the Utilities module and the Tuneable Low Pass Filter (LPF) module is used. Experimental setup with DATEx is shown in Fig. 4.

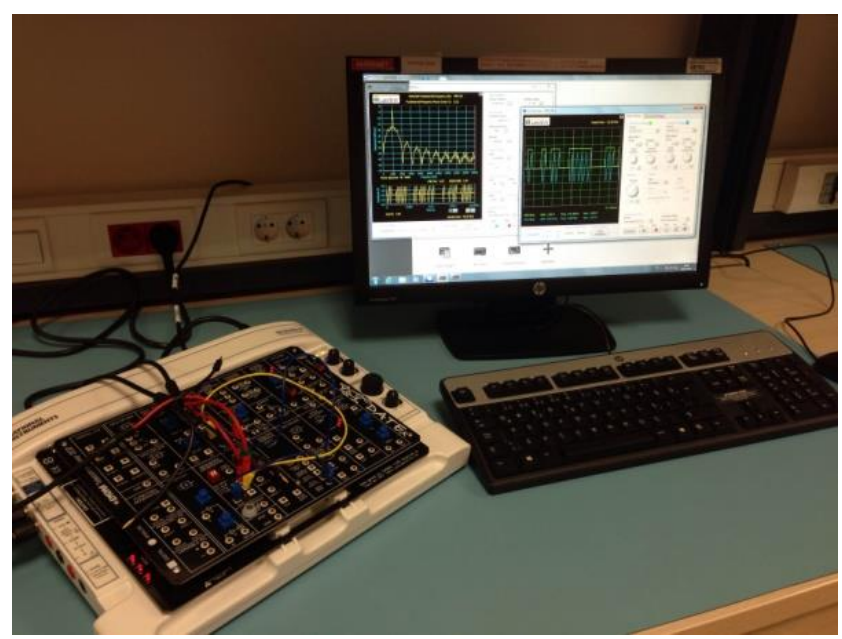

Figure 4. Experimental Setup with DATEx.

While selecting the carrier frequency there is not so many options in DATEx. In Master Signals module, signals that can be used in an implementation are; $100 \mathrm{kHz}$ sine/cosine, $2 \mathrm{kHz}$ sine, $2-8 \mathrm{kHz}$ digital. On the contrary in USRP students can use all the frequencies supported by daughterboard (400 MHz-4.4.GHz).

\section{FSK Modulation Applications}

FSK refers to a type of frequency modulation that assigns bit values to discrete frequency levels. In other words frequency does not fluctuate over symbol time but fluctuates in discrete amounts in response to message. Amplitude remains unchanged. This is a desirable characteristic for improving the power efficiency of transmitters. In FSK modulated signal consist of two different carriers;

$s 1(t)=A c \cos (2 \pi f 1 t)$

$s 2(t)=A c \cos (2 \pi f 2 t)$

One of the signal above is in response to " 0 " and the other is in response to " 1 ".

\subsection{FSK Implementation with USRP}

LabVIEW interacts with the USRP transmitter by means of four basic functions located on the block diagram's palette. Transmitter session starts with "Open Tx Session" and goes on 
with "Configure Signal" used to set parameter values in the USRP. "Write Tx Data" writes the baseband signal to the USRP for transmission and your code should be before this function. Then "Close Session" terminates transmitter operation once the while loop ends. It has five basic functions at the receiver part similarly.

While generating FSK signal, if we rewrite the frequencies as a deviation from the carrier frequency then it is called frequency deviation. This is excursion of the signal above and below the carrier frequency and indicates the quality of signal as it has particular importance in relation to bandwidth.

We can define the bandwidth of a pulse shaping filter that we create in LabVIEW as;

$B W=(1+\alpha) * R s+2 * \Delta f$

where Rs is symbol rate, $\Delta \mathrm{f}$ is frequency deviation and $\alpha$ is rolloff factor.

In the receiver part of the application beside the time and frequency domain you can use eye diagram demonstration as in Fig.5.

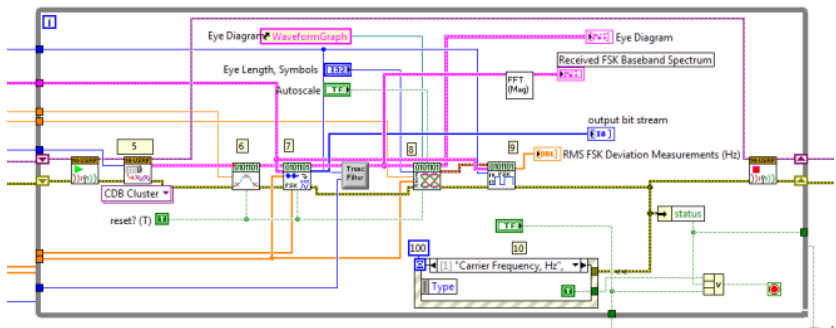

Figure 5. Block Diagram of FSK Receiver Application.

Depending the value of modulation index distortion on the received signal can be seen on the eye diagram easily.

As in Fig. 6 by changing symbol rate, alpha, filter type etc. you can easily discover conversions in the modulated signal. After mathematical theory, showing their importance on a real life signal would be very beneficial for students.

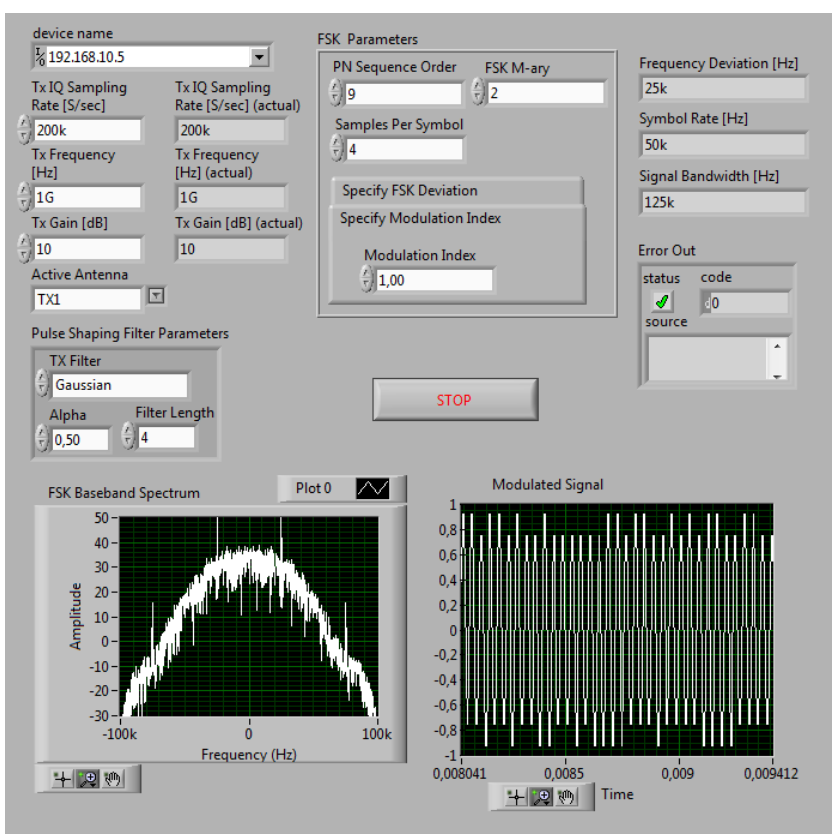

Figure 6. Front Panel of FSK Transmitter Application.

\subsection{FSK Implementation with DATEx}

For generating FSK signal in DATEx, Voltage Controlled
Oscillator (VCO) method is used. The Sequence Generator module is used for modeling the digital data and its SYNC output triggering the scope to provide a stable screeen. By using a filter we can distinguish one of the sinewave in FSK and then demodulate it with the help of a envelope detector. For this purpose Tunable LPF module is used in recovering part and then DIODE and RC LPF on the Utilities module is used to form the envelope detector. As in Fig. 7 modulation (Dotted) an demodulation (Lined) scheme of FSK is shown.

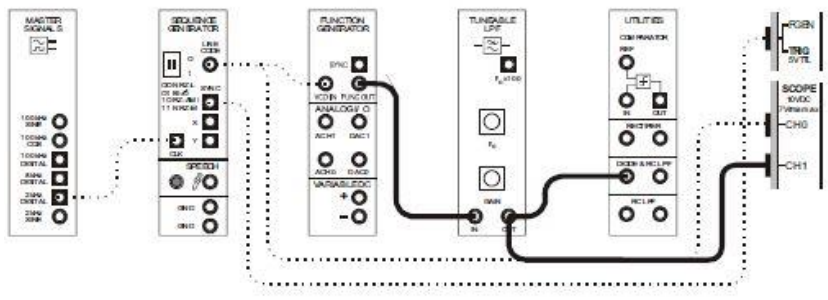

Figure 7. FSK Modulation and Demodulation Scheme.

Comparator is a useful function for restoring distorted signal. In demodulation part of the application if signal that we recover is not clear we can use comparator function in the Utilities module either.

As seen clearly from the block diagram, setting FSK demodulation is more difficult in LabVIEW than the one in DATEx. As we mentioned before some of the functions used in block diagram are standart while designing real signals. And their connections seems little bit complicated. But it is obvious that USRP gives us chance to radiate our signal in the air and demodulating/recovering a signal with additive noise is not so simple.

\section{PSK Modulation Applications}

In PSK, information is encoded on the phase of the transmitted carrier that changed between two values according to the binary signal level. When encoding bits, the phase shift could be 0 degree for encoding a " 0 ," and 180 degrees for encoding a " 1 ," or the phase shift could be -90 degrees for " 0 " and +90 degrees for a "1," thus making the representations for " 0 " and " 1 " a total of 180 degrees difference. PSK is ideal among FSK and ASK in providing the lowest average power needed for a given bit error rate. A binary PSK signal can be defined by;

$$
\begin{array}{ll}
s 1(t)=A \cos 2 \pi f c t & 0 \leq t \leq T \\
s 2(t)=-A \cos 2 \pi f c t & 0 \leq t \leq T
\end{array}
$$

These two signals have the same frequency and energy but they have a correlation coefficient of -1 .

\subsection{PSK Implementation with USRP}

Our laboratory have two USRP radios, one for transmission and the other one for reception. There are two possible configurations:

- Both radios connected to single computer (running both the transmitter and receiver VIs) with a dual-port Gigabit Ethernet interface, or using a data switch.

- Each radio connected to a different computer; one running the transmitter VI, and the other, the receiver VI.

We preferred the first one for our applications because it is better and faster for managing all the process in one screen. Our experimental set up with USRP can be seen on Fig. 9. Connection between the two USRP is provided with the Multiple Input 
Figure 11. PSK Modulation Scheme

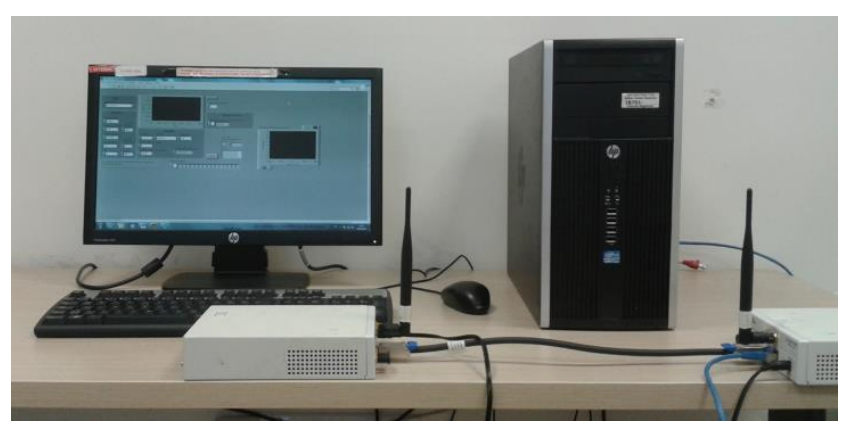

Figure 9. Experimental Setup with USRP.

Errors in communication design can be find easily using a constellation view. If the problem is related to phase, the constellation will spread around the circle. If the problem is related to magnitude, the constellation will spread off the circle, either towards or away from the origin. These graphical techniques assist in isolating problems much faster than when using other techniques. With the help of Modulation Toolkit Examples in the LabVIEW library, PSK transmitter application can be implemented easily as shown in Fig. 10.

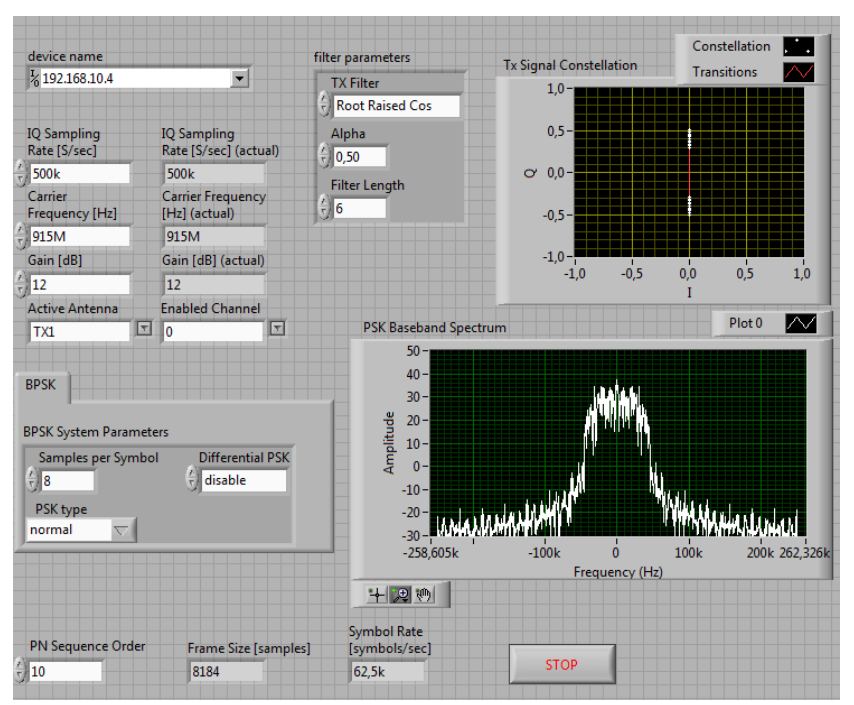

Figure 10. Front Panel of PSK Transmitter Application.

\subsection{PSK Implementation with DATEx}

Switching halves of the PSK signal's envelopes have the same shape as the message. So PSK generation and the recovery of the data can be handled by conventional Double Side Band Suppressed Carrier (DSBSC) modulation and demodulation techniques. The Sequence Generator module is used for modelling the digital data and its SYNC output triggering the scope to provide a stable screen. With the help of Multiplier module, PSK signal generated as shown in Fig. 11.

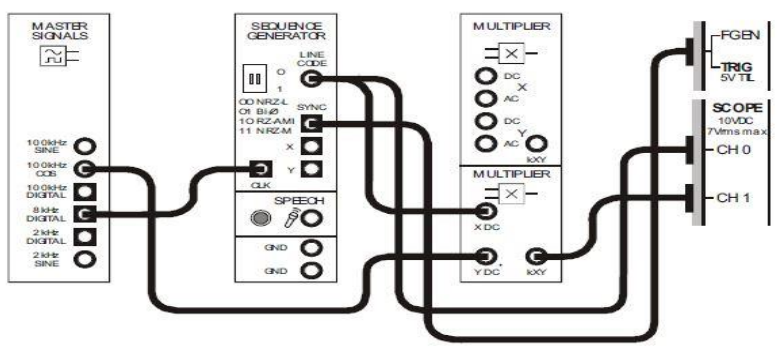

This journal is @ Advanced Technology \& Science 2013
By using second Multiplier and Tuneable LPF module we can easily carry out demodulation part of PSK signal.

\section{Conclusions}

In this study we propose a new dimension for teaching communication techniques and algorithms by focusing on two computer hosted devices, called USRP and DATEx. ASK, FSK and PSK modulation/demodulation processes presented experimentally. Our approach allows students to concentrate their efforts on real world signals by hands-on applications. Using these devices students will be able to observe signal waveforms, study the effect of noise, design components of a digital communication system. In other words replacing USRP and DATEx with the traditional hardware-only platforms will give students a chance to link the theory with practical implementations.

\section{References}

[1] S.Katz, J.Flynn, "Using Software Defined Radio (SDR) toDemonstrate Concepts In Communications and Signal Processing Courses" 39th ASEE/IEEE Frontiers in Education Conference, 2009.

[2] M. Petrova, A. Achtzehn, P. Mahonen, "System-Oriented Communications Engineering Curriculum: Teaching Design Concepts with SDR Platforms," IEEE Commun. Mag., vol. 52, no. 5, May 2014, pp. 202-09

[3] P. Goodman, "A Software-DefinedRadio Project for First YearECET Students," Proceedings of the 2007 ASEE Annual Conference.

[4] S. Guzelgoz, A. Hessam, O. Zakariaand H. Arslan, "An SDR Based Wireless Laboratory: Introducing MultiDimensional Signal Analysis" Proceeding of the SDR 2008 Technical Conference and Product Exposition.

[5] T. B. Welch, "Labview, the USRP, and Their Implications on Software Defined Radio" American Society for Engineering Education, 2011.

[6] A.L.G. Reis, A. F. B. Selva, K. G. Lenzi, S. E. Barrbin and L. G. P. Meloni, "Software Defined Radio on Digital Communications:a New Teaching Tool"Wireless and Microwave Technology Conference (WAMICON), 2012 IEEE 13th Annual, pp. 1-8.

[7] T.B. Welch and S. Shearman, "Teaching Software DefinedRadio Using the USRP and LabVIEW," IEEE Int'l. Conf.Acoustics, Speech and Signal Proc., 2012, pp. 278992.

[8] http://www.ni.com/pdf/manuals/375868a.pdf

[9] http://www.emona-tims.com/emona-product/ni-elvis-ad-onboards/ datex/

[10] H. Kyomugisha et.al, "A Remote Direct Sequence Spread Spectrum Communications Lab Utilising the Emona DATEx" Remote Engineering and Virtual Instrumentation (REV), 9th International Conference, 2012.

[11] A.Abu-aisheh, T. Eppes, A. Y. Al-Zoubi "Implementation of aRemote Analog and DigitalCommunications Lab for eLearning", IEEE EDUCON, 2010.

[12] A.Asiimwe, C. Mwikirize, P. I. Musasizi, S. S. T Togboa, A. Mwambela, "Design of a Time-Frequency Domain Analysis OnlineLaboratory Utilizing the EMONA DATEx”, IEEE AFRICON,2011. 